๑) Open Access Full Text Article

\title{
Assessment of rescue opioid use in patients with post-bunionectomy pain treated with diclofenac potassium liquid-filled capsules
}

This article was published in the following Dove Press journal:

Journal of Pain Research

4 February 2015

Number of times this article has been viewed

\author{
Joyce S Willens' \\ Iwona Bucior ${ }^{2}$ \\ Shay Bujanover ${ }^{2}$ \\ Neel Mehta ${ }^{3}$ \\ 'Villanova University, Villanova, \\ PA, ${ }^{2}$ Depomed, Inc., Newark, CA, \\ ${ }^{3}$ Weill-Cornell Pain Medicine \\ Center, New York, NY, USA
}

Correspondence: Joyce S Willens Villanova University, 800 Lancaster Ave, Villanova, PA 19085, USA

$\mathrm{Tel}+\mathrm{I} 6105196832$

Fax +16105197650

Email jwillens75@gmail.com

\begin{abstract}
When used in multimodal analgesia for acute pain, nonsteroidal anti-inflammatory drugs (NSAIDs) may reduce the requirement for opioids during the perioperative period. To provide more insight into pain treatment during the outpatient period, we examined the use of opioid rescue medication (RM) and described the relationship between pain intensity and RM use in patients with acute pain after bunionectomy. Patients received placebo or $25 \mathrm{mg}$ of a liquid-filled capsule version of the NSAID diclofenac potassium (DPLFC; $\mathrm{n}=188$ patients/group) every 6 hours during the 48-hour inpatient period through the end of outpatient dosing on day 4. Opioid RM (hydrocodone/acetaminophen tablets, $5 \mathrm{mg} / 500 \mathrm{mg}$ ) was available as needed, but taken at least 1 hour post-study medication. Fewer patients taking DPLFC versus placebo requested opioid RM during the inpatient period (4.8\%-44.7\% versus $25.0 \%-90.4 \%$ ) and also during the outpatient period $(3.7 \%-16.0 \%$ versus $13.1 \%-46.4 \%)$. Moderate or severe pain after surgery $(P=0.0307$ and $P=0.0002$, respectively) or at second dose $(P=0.0006$ and $P=0.0002$, respectively) was predictive of RM use. Patients taking RM (placebo/DPLFC) reported more adverse events (RM 55.7\%/40.6\%; no RM 29.4\%/26.0\%). Most adverse events in the RM group were opioid-related. In summary, this study shows that DPLFC lowers the requirement for opioids, which is associated with a reduction in the occurrence of treatment side effects, while maintaining adequate analgesia for patients with moderate acute pain in both the outpatient and outpatient periods. Patients with more severe pain are more likely to use RM, but they still use fewer opioids when treated with DPLFC. This suggests that multimodal treatment using DPLFC and an opioid may offer an important clinical benefit in the treatment of acute pain, including in the home environment.
\end{abstract}

Keywords: nonsteroidal anti-inflammatory drugs, diclofenac, opioids, opioid sparing, acute pain

\section{Introduction}

The pharmacologic agents most often used in the treatment of acute pain include acetaminophen (known as paracetamol outside of the USA), nonsteroidal antiinflammatory drugs (NSAIDs), and opioids. ${ }^{1,2}$ The choice between these medications is guided by the intensity of pain. Generally, acetaminophen and NSAIDs are most often used for the treatment of mild-to-moderate pain, with acetaminophen being the least potent, whereas opioids are recommended for the treatment of severe pain or when other treatments have not provided adequate pain relief. ${ }^{1,3,4}$ Despite the availability of effective treatment options, the management of acute pain is often inadequate and remains an ongoing challenge. 5,6 To address a problem of undertreated pain, the availability of opioids as the preferred treatment has expanded, but has also provided 
further opportunities for opioid abuse. ${ }^{4}$ Indeed, the misuse and diversion of prescription opioids has reached epidemic levels, ${ }^{7,8}$ with hydrocodone and oxycodone being the two most often used and abused opioids in general medicine. ${ }^{8,9}$ To resolve the issues of undertreated pain and opioid abuse, balanced pain management plans with better utilization of nonopioid agents are needed.

There is good evidence that, when used in multimodal analgesia during the perioperative period, NSAIDs may reduce the requirement for opioids, thus reducing adverse effects while providing adequate analgesic effect. ${ }^{10-13}$ However, there is little evidence for an opioid-sparing effect of NSAIDs in the treatment of acute pain in the outpatient setting. This is especially an issue because unsupervised use of opioids faces important challenges, including inappropriate use and/or overuse of opioids after discharge. In addition, patients are likely to be more active in the home environment, which may elevate pain and thus increase use of analgesics. ${ }^{14}$ Also, some patients with acute pain may use opioids for longer than is necessary and develop prolonged postoperative use of opioids after discharge. ${ }^{15}$ One barrier to dealing with these issues is our poor understanding of how effective NSAIDs are in the treatment of acute postoperative pain post discharge, and how much they may reduce the use of opioids.

A considerable number of NSAIDs with proven efficacy and safety are currently available for prescription and nonprescription treatment of acute pain, and diclofenac is one of the most widely used. ${ }^{16,17}$ A low-dose formulation of diclofenac, ie, diclofenac potassium liquid-filled capsules (DPLFCs, $25 \mathrm{mg}$; Zipsor $^{\circledR}$, Depomed, Inc., Newark, CA, USA), was developed to improve the speed and predictability of diclofenac absorption. DPLFC is approved by the US Food and Drug Administration for the treatment of mild-to-moderate acute pain in adults, and its efficacy has been demonstrated in the two most common models of acute pain, ${ }^{18}$ ie, dental pain (third molar extraction) ${ }^{19,20}$ and bunionectomy pain. ${ }^{21,22}$

The bunionectomy model of acute pain provides consistent intensity and sufficient duration of pain to permit accurate examination of single and multiple doses of an analgesic. ${ }^{18}$ Two replicate Phase III studies showed that DPLFC $25 \mathrm{mg}$ taken every 6 hours was effective in reducing post-bunionectomy pain during the inpatient and outpatient periods compared with placebo, regardless of patients' baseline pain level. ${ }^{21-23}$ DPLFC was well tolerated, and the profile of adverse events (AEs) was consistent with the known safety profiles of other NSAIDs. Throughout both bunionectomy studies, use of opioid rescue medication
(RM, hydrocodone/acetaminophen tablets) was allowed and documented. To comprehensively characterize the use of opioid RM in patients with post-bunionectomy pain treated with DPLFC versus placebo during the inpatient and outpatient periods, and to describe the relationship between pain intensity and opioid RM use, we analyzed pooled data from the two Phase III studies.

\section{Patients and methods}

\section{Patients}

Patients aged 18-65 years who underwent primary unilateral first metatarsal bunionectomy with or without hammertoe repair and internal fixation and had a pain intensity score at rest of $\geq 4$ on a $0-10$ numerical pain rating scale (NPRS; where $0=$ no pain and $10=$ worst possible pain) were enrolled in the studies. Patients were excluded if, following surgery up to the initial dose of study medication, they had taken NSAIDs or opioids that were not specified in the protocol. Patients were also excluded if they had a known allergy or hypersensitivity to diclofenac or other NSAIDs, aspirin, opioids (including hydrocodone or codeine), or acetaminophen. Detailed patient inclusion/exclusion criteria have been previously reported. ${ }^{21,22}$ All patients provided their written informed consent prior to screening.

\section{Study design and treatment}

Two multicenter, double-blind, parallel-group, placebocontrolled studies of the analgesic effect of DPLFC in patients with pain following bunionectomy were conducted concurrently from August 2006 to January 2007 at ten investigational sites in the USA. ${ }^{21,22}$ Both studies consisted of two inpatient dosing periods: a single-dose period on the morning of day 1 and a 48-hour multiple-dose period that began with the second dose of study drug on day 1 and continued through dose 10 on the morning of day 3 (Figure 1). The outpatient dosing period began upon patient discharge on the morning of day 3 , continued through the end of day 4 when patients took the last dose of study medication, and ended on the morning of day 5 (or upon early termination) when patients made the final assessment of their pain intensity on the NPRS and returned to the clinic for the final visit.

Surgery on day 0 was performed using Mayo block of the first metatarsal with local anesthesia, propofol for intravenous sedation during surgery, and if clinically indicated, fentanyl, morphine, or midazolam. Following surgery, patients received routine standard of care, ie, use of ice packs was allowed on days 0 and 1 , but was discontinued at least 1 hour before randomization and not permitted 


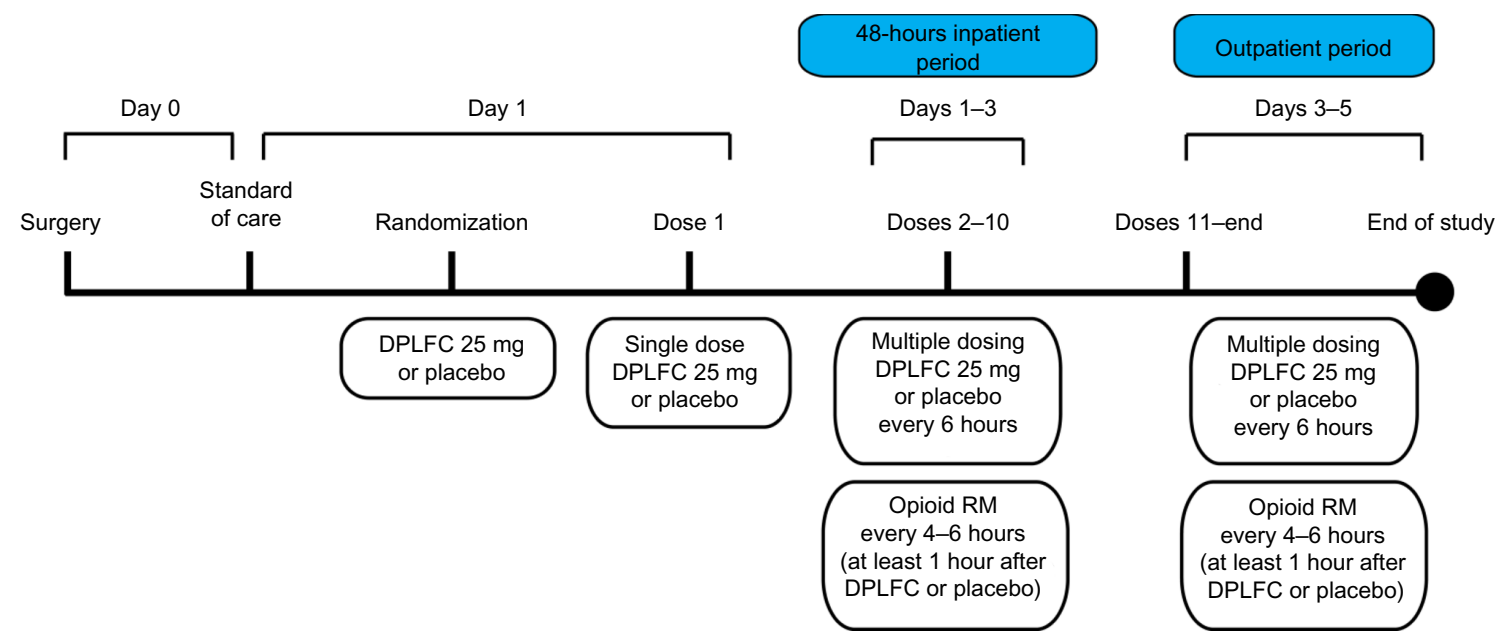

Figure I Study design.

Notes: After surgery, patients received standard care for analgesia (hydrocodone $5 \mathrm{mg} /$ acetaminophen $500 \mathrm{mg}$ and ice packs; stopped before randomization), and were required to achieve a qualifying level of pain ( $\geq 4$ on the $0-10$ NPRS) before randomization to receive DPLFC 25 mg or matching placebo. Eight hours after the initial single dose of study medication, patients received the second dose, which marked the beginning of the 48-hour inpatient period (days I-3). The outpatient period started upon discharge on day 3 and ended on the morning of day 5 . Throughout the inpatient and outpatient periods, patients received DPLFC 25 mg every 6 hours, and opioid RM (hydrocodone $5 \mathrm{mg} /$ acetaminophen $500 \mathrm{mg}, \mathrm{I}-2$ tablets) every 4-6 hours as needed, but at least I hour after receiving the study medication.

Abbreviations: NPRS, numerical pain rating scale; DPLFC, diclofenac potassium liquid-filled capsule; RM, rescue medication.

thereafter (Figure 1). Hydrocodone $5 \mathrm{mg}$ /acetaminophen $500 \mathrm{mg}$ (one to two tablets every 4-6 hours) was permitted for analgesia but was to be discontinued $\geq 4$ hours prior to randomization.

The next morning (day 1), patients with a pain intensity score at rest (no activity of the affected toe for $\geq 10$ minutes prior to assessment) of $\geq 4$ on the NPRS were randomized to receive DPLFC $25 \mathrm{mg}$ or matching placebo (Figure 1). The second dose of study medication, which marked the beginning of the 48-hour inpatient period, was administered 8 hours after the initial single dose or earlier if the patient was not experiencing adequate pain relief (patients were encouraged to delay the second dose of study drug until 1 hour after the first dose). Following the second dose, patients received the study medication every 6 hours throughout the inpatient period (no more than four doses in 24 hours were allowed). During this period, patients could request opioid RM (the same medication that was used before randomization): hydrocodone $5 \mathrm{mg} /$ acetaminophen $500 \mathrm{mg}$, one to two tablets every 4-6 hours as needed, with a maximum of eight tablets per day (patients were encouraged to wait at least 1 hour after receiving the study medication before requesting opioid $\mathrm{RM}$ ). Patients who took opioid RM recorded their pain intensity and the number of tablets taken. Subsequent doses of study medication were taken on schedule.

After dose 10 of the study medication, on the morning of day 3, patients were discharged from the clinic (start of the outpatient period), and self-administered the study medication every 6 hours until the end of day 4 (Figure 1).
Patients were provided with opioid RM to be taken on the same schedule as during the inpatient period.

\section{Assessments of efficacy and safety}

Efficacy assessments included pain intensity and use of opioid RM. Pain intensity scores using the NPRS were obtained at the time of study drug administration (ie, every 6 hours), 3 hours after study drug administration, and at the time of any administration of opioid RM. Baseline pain intensity was the last pain intensity score obtained before study drug dosing on day 1 . For the use of opioid RM, the number of patients, time, and number of tablets for each dose was determined. Assessments were transcribed onto the case report form during the 48-hour inpatient period, and recorded via an interactive voice response system during the outpatient period. If patients failed to record their information within the specified time period, the interactive voice response system telephoned them and provided a recorded reminder that study information had not been reported. The final NPRS assessment via the interactive voice response system was performed on the morning of day 5 .

Safety assessments included the incidence and severity of AEs and serious AEs, analysis of discontinuations due to AEs, clinical laboratory assessments of serum chemistry and hematology, vital signs, and findings on physical examination. AEs were collected from the time of randomization throughout the study, with the last assessment on day 5 when patients returned to the clinic for the final visit. All AEs were classified according to the System Organ Class and Preferred Term using the 
Medical Dictionary for Regulatory Activities $\left(\right.$ MedDRA ${ }^{\circledR}$ ). Baseline safety assessments were performed at the screening visit, within the 21 days prior to surgery.

\section{Statistical analysis}

Data from two studies of the analgesic effect of DPLFC in patients with post-bunionectomy pain were integrated prior to this analysis. All patients who received study drug, regardless of whether they recorded a post-dose NPRS score, and who had no major protocol violation that could influence efficacy were included in the efficacy population. The safety population included all patients who received study drug. For the analyses, patients were assigned to one of two subgroups: patients who took opioid RM at any time of the study and patients who did not take opioid RM. For analysis of predictive factors for using opioid RM, patients were assigned to two subgroups: patients with a baseline NPRS score of 4-6 (moderate pain) and patients with a baseline NPRS score of 7-10 (severe pain); there were no patients with a baseline NPRS score of 1-3 (mild pain).

The proportion of patients who used versus did not use opioid RM was analyzed using the Cochran-MantelHaenszel test with pooled site as strata. One-way analysis of variance with treatment as a factor was used to analyze number of administrations and amount of opioid RM. The Cochran-Mantel-Haenszel test with pooled site as strata was used to analyze NPRS scores at various time points for patients who used opioid RM versus those who did not use opioid RM during the 48-hour inpatient period. For analysis of predictive factors, the odds ratio (OR) estimates, confidence intervals (CIs), and $P$-values were from a logistic regression model for the event of using opioid RM. Independent variables in the model were age, sex, race, pain score at baseline, change in pain score from baseline to dose 2 , duration of surgery, and time from end of surgery to dosing. Statistical significance was set at $P \leq 0.05$.

Descriptive statistics were used to summarize baseline demographic and disease characteristics. For analysis of AEs, patients who had more than one AE within a System Organ Class and patients who had more than one AE assigned to the same Preferred Term were counted once. For analysis of AEs per day, these patients were counted once per day of occurrence.

\section{Results}

\section{Patients}

A total of 376 patients with post-bunionectomy pain who received DPLFC $25 \mathrm{mg}(\mathrm{n}=188)$ or matching placebo $(n=188)$ were included in the efficacy analyses. The safety analyses included 200 patients in the placebo group and 201 patients in the DPLFC group. Patient demographics and baseline disease characteristics were similar between the DPLFC and placebo groups (Table 1). Before randomization to receive DPLFC $25 \mathrm{mg}$ or matching placebo, almost all patients $(99.5 \%$ ) used opioid RM (stopped $\geq 4$ hours before randomization), and the majority used ice packs $(75.8 \%$; stopped at least 1 hour before randomization). The mean baseline pain score was 7.3 , with $66.8 \%$ of patients reporting severe (NPRS score $\geq 7$ ) pain at baseline, and $33.2 \%$ of patients reporting moderate (NPRS score 4-6) pain at baseline (Table 1).

\section{Use of rescue medication}

After surgery, during both the inpatient and outpatient periods, significantly fewer patients treated with DPLFC requested opioid RM compared with patients treated with

Table I Demographics and baseline disease characteristics

\begin{tabular}{|c|c|c|c|}
\hline & $\begin{array}{l}\text { Placebo } \\
(n=188)\end{array}$ & $\begin{array}{l}\text { DPLFC } 25 \mathrm{mg} \\
(\mathrm{n}=188)\end{array}$ & $\begin{array}{l}\text { Total } \\
(\mathrm{N}=376)\end{array}$ \\
\hline \multicolumn{4}{|l|}{ Age (years) } \\
\hline Mean (SD) & $42.7(12.2)$ & $43.3(12.2)$ & $43.0(12.2)$ \\
\hline Range & $18-65$ & $18-65$ & $18-65$ \\
\hline \multicolumn{4}{|l|}{ Sex, n (\%) } \\
\hline Female & I6I (85.6) & $162(86.2)$ & $323(85.9)$ \\
\hline Male & $27(\mid 4.4)$ & $26(13.8)$ & $53(14.1)$ \\
\hline \multicolumn{4}{|l|}{ Race, n (\%) } \\
\hline Caucasian & $103(54.8)$ & $117(62.2)$ & $220(58.5)$ \\
\hline Hispanic or Latino & $40(21.3)$ & $33(17.6)$ & $73(19.4)$ \\
\hline Black & $34(18.1)$ & $29(15.4)$ & $63(16.8)$ \\
\hline Asian & $7(3.7)$ & $6(3.2)$ & $13(3.5)$ \\
\hline Other & $4(2.1)$ & $3(1.6)$ & $7(1.9)$ \\
\hline \multicolumn{4}{|l|}{ Weight (kg) } \\
\hline Mean (SD) & $73.2(13.5)$ & $71.7(14.0)$ & $72.5(13.8)$ \\
\hline \multicolumn{4}{|c|}{ Duration of surgery (minutes) } \\
\hline Mean (SD) & $33.5(13.4)$ & $33.9(15.4)$ & 33.7 (I4.4) \\
\hline \multicolumn{4}{|c|}{ Time from end of surgery to dosing (hours) } \\
\hline Mean (SD) & $19.5(2.4)$ & $19.5(2.5)$ & $19.5(2.5)$ \\
\hline \multicolumn{4}{|c|}{ Time from transfer to unit until dosing (hours) } \\
\hline Mean (SD) & $18.9(2.4)$ & $19.0(2.5)$ & $18.9(2.5)$ \\
\hline $\begin{array}{l}\text { Use of ice packs before } \\
\text { randomization, } \mathrm{n}(\%)\end{array}$ & $140(74.5)$ & I $45(77.1)$ & $285(75.8)$ \\
\hline $\begin{array}{l}\text { Use of hydrocodone before } \\
\text { randomization, } \mathrm{n}(\%)\end{array}$ & $188(100)$ & $186(98.9)$ & $374(99.5)$ \\
\hline \multicolumn{4}{|l|}{ Baseline pain score } \\
\hline Mean (SD) & $7.3(1.7)$ & $7.2(1.7)$ & $7.3(1.7)$ \\
\hline Range & $4-10$ & $4-10$ & $4-10$ \\
\hline \multicolumn{4}{|c|}{ Baseline pain score by category, n (\%) } \\
\hline Mild & 0 & 0 & 0 \\
\hline Moderate & $57(30.3)$ & $68(36.2)$ & $125(33.2)$ \\
\hline Severe & $|3|(69.7)$ & $120(63.8)$ & $25 \mathrm{I}(66.8)$ \\
\hline
\end{tabular}

Abbreviations: SD, standard deviation; DPLFC, diclofenac potassium liquid-filled capsule. 


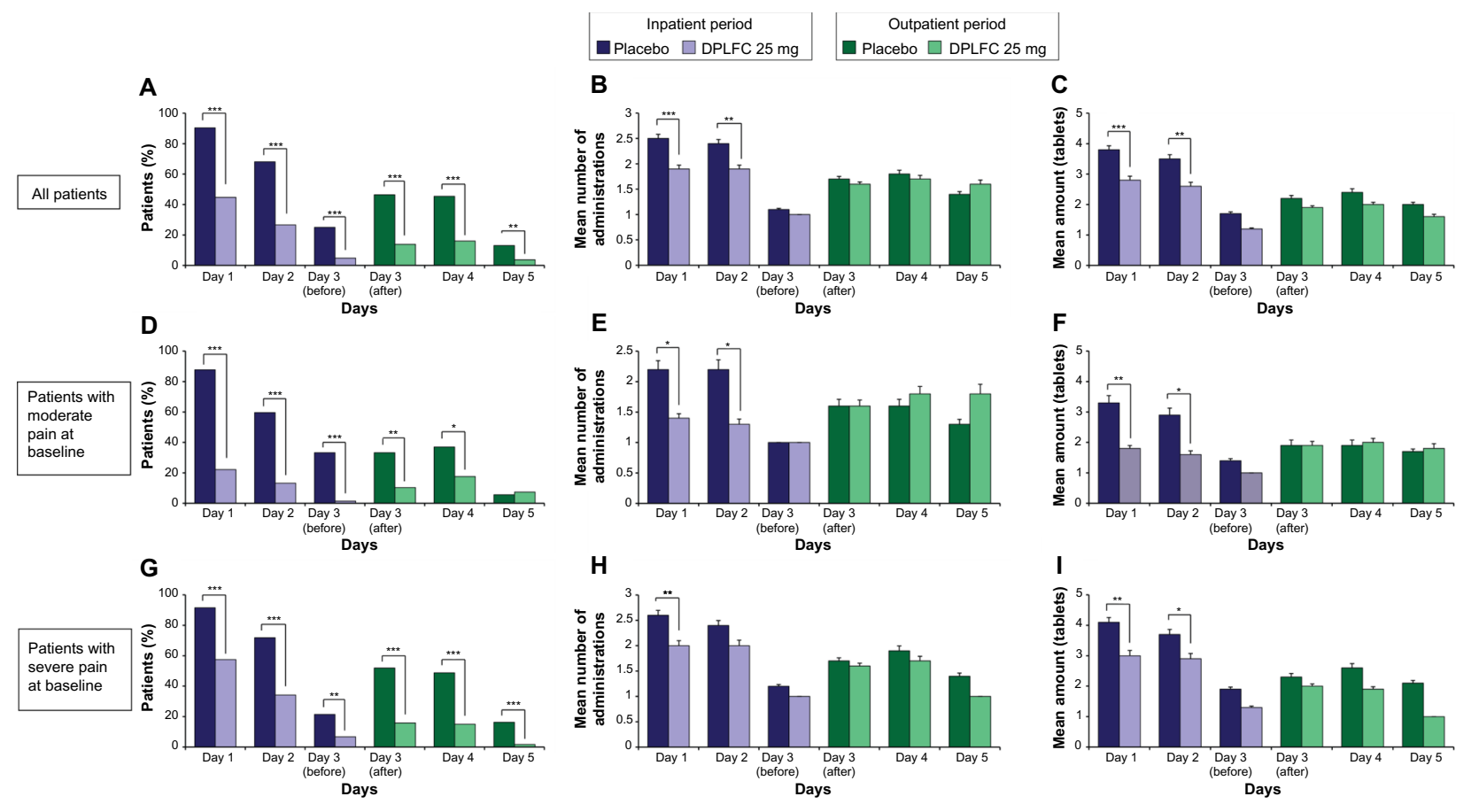

Figure 2 Summary of opioid RM use.

Notes: Throughout the inpatient (days I-3) and outpatient (days 3-5) periods, patients received DPLFC every 6 hours and, if needed, opioid RM every 4-6 hours, but at least I hour after receiving the study medication. The use of opioid RM (A, D, and $\mathbf{G})$ was analyzed using Cochran-Mantel-Haenszel. The percentage of all patients (A), patients with moderate pain at baseline (D), and patients with severe pain at baseline (G) in the placebo or DPLFC group who used or did not use opioid RM. For patients who used opioid RM, the number of administrations (B, E, and $\mathbf{H}$ ) and amount of tablets (C, F, and I) were analyzed using one-way analysis of variance. Mean (SEM) number of administrations of opioid RM by all patients (B), patients with moderate pain at baseline (E), and patients with severe pain at baseline (H). Mean (SEM) amount of opioid RM (tablets) for all patients (C), patients with moderate pain at baseline (F), and patients with severe pain at baseline (I). $* P<0.05 ; * * P<0.005$; $* * * P<0.000$ I.

Abbreviations: RM, rescue medication; DPLFC, diclofenac potassium liquid-filled capsule; SEM, standard error of the mean.

placebo (Figure 2A). The use of opioid RM increased on day 3 after discharge compared with day 3 before discharge (placebo, $46.4 \%$ versus $25.0 \%$; DPLFC, $13.8 \%$ versus $4.8 \%$ ); however, patients treated with DPLFC continued to use less opioid RM during the outpatient period compared with patients treated with placebo. For patients grouped by intensity of pain at baseline, fewer patients with moderate (Figure 2D) or severe (Figure 2G) pain at baseline treated with DPLFC requested opioid RM during the inpatient and outpatient periods compared with patients treated with placebo. More DPLFC patients with severe pain at baseline versus patients with moderate pain at baseline requested opioid RM during the inpatient period $(57.5 \%$ versus $22.1 \%$ on day $1 ; 34.2 \%$ versus $13.2 \%$ on day $2 ; 6.7 \%$ versus $1.5 \%$ on day 3), but the use of opioid RM was similar between these patients during the outpatient period (comparing Figure 2D and G). Use of opioid RM by patients with moderate pain at baseline treated with DPLFC, but not placebo, increased after discharge compared with day 3 before discharge (Figure 2D). The use of opioid RM by patients with severe pain at baseline increased for both the DPLFC and placebo groups during days 3 and 4 of the outpatient period (Figure 2G).
Among patients who requested opioid RM, the number of administrations (Figure 2B) and amount of RM tablets (Figure 2C) were lower for patients treated with DPLFC versus placebo only on days 1 and 2 . Similar results were observed for patients with moderate (Figure 2E and F) or severe (Figure 2H and I) pain at baseline.

\section{Pain intensity during the 48-hour inpatient period}

During the inpatient period, the majority of patients treated with placebo (173/188; 92\%) requested opioid RM, and experienced significantly higher mean pain intensity on the NPRS at baseline compared with patients who did not request opioid RM (7.4 versus 6.3, respectively, $P=0.0207$; Figure 3A). Similarly, patients treated with DPLFC and who used opioid RM reported higher pain intensity at baseline compared with those who did not use opioid RM (7.9 versus 6.5 , respectively; $P<0.0001$; Figure 3B). In contrast with patients treated with placebo, approximately half of those treated with DPLFC $(92 / 188 ; 49 \%)$ requested opioid RM.

Over time, patients in the placebo group using opioid $\mathrm{RM}(\mathrm{n}=173)$ experienced a decrease in mean pain intensity 

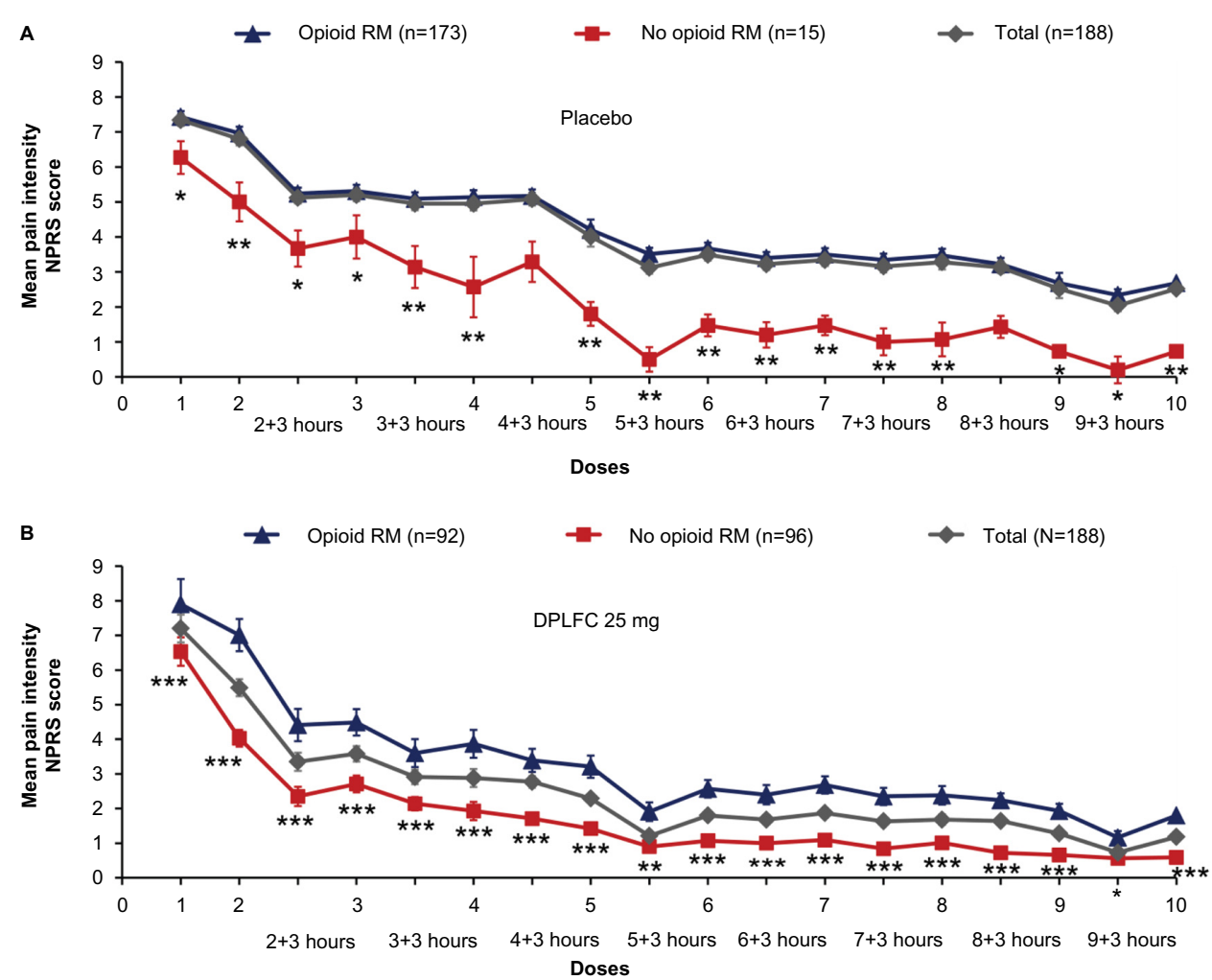

Figure 3 Mean pain intensity on the NPRS over time during the inpatient period.

Notes: At the initial, single dose (dose I) and during the 48-hour inpatient period (doses I-10), patients received DPLFC every 6 hours and, if needed, opioid RM every $4-6$ hours, but at least I hour after receiving the study medication. Pain intensity scores were assessed using the NPRS. Mean (SEM) pain intensity scores at the time of study drug administration (ie, every 6 hours) and 3 hours after study drug administration for patients in the placebo group (A) and for patients in the DPLFC group (B) who used or did not use opioid RM. P-values for the difference NPRS scores between patients who used opioid RM versus patients who did not use opioid RM were calculated using the Cochran-Mantel-Haenszel test. $* P<0.05$; ** $P<0.005$; *** $P<0.0001$.

Abbreviations: NPRS, numerical pain rating scale; RM, rescue medication; DPLFC, diclofenac potassium liquid-filled capsule; SEM, standard error of the mean.

from 7.4 at dose 1 to 2.7 at the end of the 48 -hour inpatient period (dose 10), with a mean reduction of -4.8 from dose 1 to dose 10 (Figure 3A). Patients not using opioid RM (n=15), reported a decrease in mean pain intensity from 6.3 to 0.7 , with a mean reduction of -5.5 (Figure $3 \mathrm{~A}$ ). The difference in mean pain reduction between patients in the placebo group using opioid RM and patients not using opioid RM was not statistically significant. Because of the difference in patient populations ( $n=173$ versus $n=15$ ), the graph representing the change in pain intensity for the total patient population $(n=188)$ was skewed toward the graph representing the change in pain intensity for patients who requested opioid RM $(\mathrm{n}=173$; Figure 3A). Among patients treated with DPLFC, those using opioid $\mathrm{RM}(\mathrm{n}=92)$ reported a decrease in mean pain intensity from 7.9 at dose 1 to 1.8 at dose 10 (Figure $3 \mathrm{~B})$, with a mean reduction of -6.1 . DPLFC patients not using opioid $\mathrm{RM}(\mathrm{n}=96)$ experienced a decrease in mean pain intensity from 6.5 to 0.6 (mean reduction, -5.9 ). Overall, the difference in pain reduction from dose 1 to dose 10 between patients using opioid RM and patients not using opioid RM was not statistically significant. In contrast with patients in the placebo group, similar numbers of patients treated with DPLFC did not request opioid RM versus requested opioid $\mathrm{RM}$ ( $\mathrm{n}=96$ versus $\mathrm{n}=92$ ), and thus the graph representing the change in pain intensity for the total patient population $(n=188)$ was not skewed toward any of the two graphs representing patient subgroups (Figure 3B).

To analyze changes in pain intensity between patients treated with an opioid versus with an opioid and DPLFC, patients in the placebo group who used opioid RM $(173 / 188 ; 92 \%)$ and patients in the DPLFC group who used opioid RM (92/188; $49 \%$ ) were compared. There was a trend toward a greater change in pain intensity over time for patients treated with both DPLFC and opioid RM compared with patients treated with the opioid RM alone (compare Figure 2A and B), and the difference between mean changes from dose 1 to dose 10 ( -6.10 versus -4.75 , respectively) was statistically significant $(P<0.01)$.

\section{Risk factors for RM use}

Multivariate regression analyses were performed to identify risk factors for using opioid RM during the inpatient and outpatient periods. For all patients treated with placebo 
(although the majority of these patients were using opioid RM), significant predictors of opioid RM use were female sex (although with a wide 95\% CI), baseline pain score, and change in pain score from baseline to dose 2 (Table 2). When grouped by pain intensity at baseline, there were no significant predictive factors for patients with moderate baseline pain scores. For patients with severe baseline pain scores, predictive factors were non-Caucasian race (with a wide $95 \% \mathrm{CI}$ ), baseline pain score (with a wide $95 \% \mathrm{CI}$ ), and change in pain score from baseline to dose 2 . In contrast, for patients treated with DPLFC, analysis of predictive factors revealed more consistent results. Baseline pain scores and change from baseline to dose 2 in pain score were the only significant predictors for using opioid RM in all patients treated with DPLFC and in patients with moderate or severe baseline pain scores (Table 2).

\section{Safety}

Consistent with the analysis in the efficacy population, the majority of patients in the safety population who received placebo requested opioid RM ( $n=183$ for patients who

Table 2 Predictive factors for opioid RM use

\begin{tabular}{|c|c|c|c|}
\hline Predictive factor & OR & $95 \% \mathrm{Cl}$ & $P$-value \\
\hline \multicolumn{4}{|l|}{ Patients treated with placebo } \\
\hline \multicolumn{4}{|l|}{ Total } \\
\hline Sex (female versus male) & 6.134 & $1.566-24.027$ & 0.0092 \\
\hline Baseline pain score & 1.862 & $1.202-2.883$ & 0.0054 \\
\hline $\begin{array}{l}\text { Change in pain score from } \\
\text { baseline to dose } 2\end{array}$ & 1.398 & $1.037-1.885$ & 0.0278 \\
\hline \multicolumn{3}{|c|}{ Patients with moderate baseline pain scores } & \\
\hline \multicolumn{4}{|c|}{ Patients with severe baseline pain scores } \\
\hline $\begin{array}{l}\text { Race (non-Caucasian versus } \\
\text { Caucasian) }\end{array}$ & 0.092 & $0.010-0.857$ & 0.0362 \\
\hline Baseline pain score & 8.157 & $1.395-47.700$ & 0.0198 \\
\hline $\begin{array}{l}\text { Change in pain score from } \\
\text { baseline to dose } 2\end{array}$ & 1.654 & $1.024-2.672$ & 0.0398 \\
\hline \multicolumn{4}{|c|}{ Patients treated with DPLFC 25 mg } \\
\hline \multicolumn{4}{|c|}{ Total } \\
\hline Baseline pain score & 1.977 & $1.538-2.542$ & $<0.0001$ \\
\hline $\begin{array}{l}\text { Change in pain score from } \\
\text { baseline to dose } 2\end{array}$ & 1.506 & I.279-1.772 & $<0.0001$ \\
\hline \multicolumn{4}{|c|}{ Patients with moderate baseline pain scores } \\
\hline Baseline pain score & 3.458 & I.I22-10.654 & 0.0307 \\
\hline $\begin{array}{l}\text { Change in pain score from } \\
\text { baseline to dose } 2\end{array}$ & 2.120 & $1.38 \mathrm{I}-3.254$ & 0.0006 \\
\hline \multicolumn{4}{|c|}{ Patients with severe baseline pain scores } \\
\hline Baseline pain score & 2.791 & $1.613-4.829$ & 0.0002 \\
\hline $\begin{array}{l}\text { Change in pain score from } \\
\text { baseline to dose } 2\end{array}$ & 1.445 & $1.191-1.752$ & 0.0002 \\
\hline
\end{tabular}

Abbreviations: $\mathrm{OR}$, odds ratio; $\mathrm{Cl}$, confidence interval; $\mathrm{RM}$, rescue medication; DPLFC, diclofenac potassium liquid-filled capsule. used opioid RM versus $\mathrm{n}=17$ for patients who did not use opioid RM; Table 3). For patients in the safety population who received DPLFC, 101 used opioid RM and 100 did not (Table 3).

For both the placebo and DPLFC groups, patients who took opioid RM throughout the inpatient and outpatient periods experienced more AEs compared with patients who did not take opioid RM (Table 3). For the placebo group, 55.7\% of patients taking opioid RM reported at least one AE compared with $29.4 \%$ of those not taking opioid RM. Consistent with this, for the DPLFC group, $40.6 \%$ of patients taking opioid RM reported at least one AE compared with $26.0 \%$ of patients not taking opioid RM. For the placebo group, AEs experienced more often by patients using opioid RM versus not using opioid RM included nausea $(24.0 \%$ versus $5.9 \%$, respectively), vomiting ( $8.7 \%$ versus $5.9 \%)$, headache $(8.2 \%$ versus $5.9 \%$ ), and constipation (3.8\% versus $0 \%$; Table 3 ). For the DPLFC group, the AEs experienced most frequently by patients using opioid RM versus not using opioid RM included nausea (20.8\% versus $5.0 \%)$, vomiting (10.9\% versus $1.0 \%)$, headache $(9.9 \%$ versus $3.0 \%)$, dizziness $(5.9 \%$ versus $1.0 \%$ ), constipation (5.9\% versus $2.0 \%$ ), and somnolence $(4.0 \%$ versus $2.0 \%)$.

The incidence of AEs decreased over time for all patients (Figure 4). For the total patient population, the percentage of patients reporting any $\mathrm{AE}$ decreased from $35.5 \%$ on day 1 to $7.0 \%$ on day 5 for the placebo group, and from $21.9 \%$ to $1.5 \%$ for the DPLFC group (Figure 4A). For patients taking opioid RM, the percentage of patients reporting AEs decreased from $37.7 \%$ to $7.7 \%$ for the placebo group, and from $26.7 \%$ to $2.0 \%$ for the DPLFC group (Figure 4B). For patients not taking opioid $\mathrm{RM}$, the proportion of patients decreased from $11.8 \%$ to no patients reporting any $\mathrm{AE}$ for the placebo group, and from $17.0 \%$ to $1.0 \%$ for the DPLFC group (Figure 4C).

Two patients discontinued the study due to an $\mathrm{AE}$ (one patient treated with placebo with severe muscle spasms deemed unrelated to study drug and another patient treated with DPLFC who developed a mild rash that was deemed possibly related to study drug). Two serious AEs (severe knee pain and left leg deep vein thrombosis) were reported, both in patients treated with placebo and taking opioid RM, and neither was considered related to the study drug (Table 3).

\section{Discussion}

Although opioids are associated with risks for abuse, addiction, and diversion that have resulted in tighter legal 
Table 3 Summary of treatment-emergent adverse events

\begin{tabular}{|c|c|c|c|c|c|c|}
\hline & \multicolumn{2}{|c|}{ Opioid RM } & \multicolumn{2}{|c|}{ No opioid RM } & \multicolumn{2}{|l|}{ Total } \\
\hline & $\begin{array}{l}\text { Placebo } \\
(n=\mid 83)\end{array}$ & $\begin{array}{l}\text { DPLFC } 25 \mathrm{mg} \\
(\mathrm{n}=10 \mathrm{I})\end{array}$ & $\begin{array}{l}\text { Placebo } \\
(n=17)\end{array}$ & $\begin{array}{l}\text { DPLFC } 25 \mathrm{mg} \\
(\mathrm{n}=100)\end{array}$ & $\begin{array}{l}\text { Placebo } \\
(\mathrm{N}=\mathbf{2 0 0})\end{array}$ & $\begin{array}{l}\text { DPLFC } 25 \mathrm{mg} \\
(\mathrm{N}=20 \mathrm{I})\end{array}$ \\
\hline Patients with $\geq I A E$ & $102(55.7)$ & $41(40.6)$ & $5(29.4)$ & $26(26.0)$ & $107(53.5)$ & $67(33.3)$ \\
\hline Patients with $\geq I$ SAE & $2(1.1)$ & 0 & 0 & 0 & $2(1.0)$ & 0 \\
\hline \multicolumn{7}{|c|}{ Most common AEs*, n (\%) } \\
\hline Nausea & $44(24.0)$ & $21(20.8)$ & I (5.9) & $5(5.0)$ & $45(22.5)$ & $26(12.9)$ \\
\hline Vomiting & $16(8.7)$ & II (10.9) & I (5.9) & $\mathrm{I}(\mathrm{I} .0)$ & $17(8.5)$ & $12(6.0)$ \\
\hline Headache & $15(8.2)$ & $10(9.9)$ & I (5.9) & $3(3.0)$ & $16(8.0)$ & $13(6.5)$ \\
\hline Dizziness & II (6.0) & $6(5.9)$ & I (5.9) & $\mathrm{I}(\mathrm{I} .0)$ & $12(6.0)$ & $7(3.5)$ \\
\hline Constipation & $7(3.8)$ & $6(5.9)$ & 0 & $2(2.0)$ & $7(3.5)$ & $8(4.0)$ \\
\hline Abdominal pain & $2(1.1)$ & $4(4.0)$ & I (5.9) & $5(5.0)$ & $3(1.5)$ & $9(4.5)$ \\
\hline Pyrexia & $8(4.4)$ & 0 & 0 & 0 & $8(4.0)$ & 0 \\
\hline Somnolence & $5(2.7)$ & $4(4.0)$ & 0 & $2(2.0)$ & $5(2.5)$ & $6(3.0)$ \\
\hline Diarrhea & $5(2.7)$ & 0 & 0 & $3(3.0)$ & $5(2.5)$ & $3(1.5)$ \\
\hline Pruritus & $5(2.7)$ & I (I.0) & 0 & $2(2.0)$ & $5(2.5)$ & $3(1.5)$ \\
\hline Hyperhidrosis & $4(2.2)$ & I (I.0) & 0 & 0 & $4(2.0)$ & $\mathrm{I}(0.5)$ \\
\hline Anxiety & $4(2.2)$ & I (I.0) & 0 & 0 & $4(2.0)$ & $\mathrm{I}(0.5)$ \\
\hline
\end{tabular}

Note: *Occurring in $\geq 2 \%$ of patients.

Abbreviations: AE, adverse event; SAE, serious adverse event; RM, rescue medication; DPLFC, diclofenac potassium liquid-filled capsule.

regulations regarding their prescription and distribution, their use, especially in outpatient settings, continues to increase..$^{24,25}$ Concurrently, opioid-related overdoses and deaths remain a widespread concern. ${ }^{26}$ Thus, better management of acute pain exploiting nonopioid agents with distinct mechanisms of action, efficacy, and safety is desirable.

NSAIDs, which are very effective on somatic and breakthrough pain, are one of the most common nonopioid analgesics used for management of postoperative pain. . 12,13,27 $^{2}$ Although NSAIDs do not provide sufficient analgesia for severe pain, they are one of the primary agents recommended for multimodal management of acute pain, which includes administration of two or more drugs that act by different mechanisms. ${ }^{28}$ When used in multimodal treatment during the perioperative period, NSAIDs can reduce the use of opioids, can potentially improve analgesia, and/or reduce opioidrelated adverse effects. ${ }^{11,29,30}$ Moreover, the anti-inflammatory effect of NSAIDs provides additional benefit for multimodal use with opioids in the short-term management of severe acute pain. ${ }^{12}$ To better understand the use of NSAIDs and their potential opioid-sparing effect in the treatment of acute pain in the outpatient setting, the current study analyzed the outcomes of treatment of acute, post-bunionectomy pain with DPLFC before and after discharge.

DPLFC alone appeared to be sufficient to provide adequate pain relief for patients with moderate pain at baseline during both the inpatient and outpatient periods. When used with opioid RM, it provided a significantly greater reduction in pain intensity over time when compared with opioid RM used alone by patients in the placebo group. Significantly fewer patients treated with DPLFC requested opioid RM compared with patients treated with placebo during both the inpatient and outpatient periods. Patients who reported slightly more severe pain at baseline were more likely to request an opioid RM, and continued to use it although their pain intensity scores diminished to moderate and mild levels over time. Consistent with these observations, pain intensity at baseline and early pain relief (change in pain intensity from baseline to dose 2) were significant predictors of using opioid RM in patients treated with DPLFC. Predictors of opioid use for patients treated with placebo included pain intensity scores as well as patient demographics (female sex and non-Caucasian race). However, most of these predictors, especially patient demographics, were not consistent for all patients versus patients divided by the severity of their pain, and had a wide $95 \% \mathrm{CI}$, indicating a low confidence level of these predictors and thus little knowledge about their true effect on opioid use by patients in the placebo group. Together, these results indicate that pain intensity is the key driver for use of opioid RM in patients with acute pain, and patients with moderate pain can receive adequate pain treatment with an NSAID alone. The ability of individual patients to tolerate pain and/or what type of analgesic they are accustomed to are important factors, since many patients continued using opioid RM over time, even though their pain intensity levels decreased to mild levels. Better patient education, especially in regard to self-treatment after discharge, will 


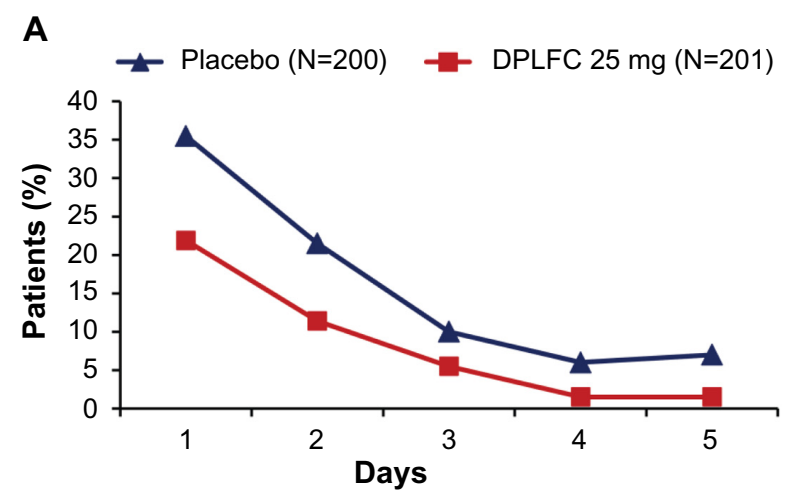

B

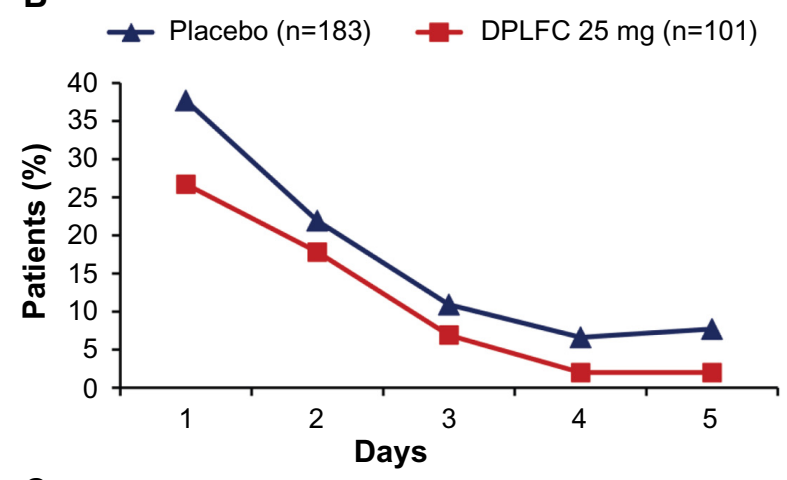

c

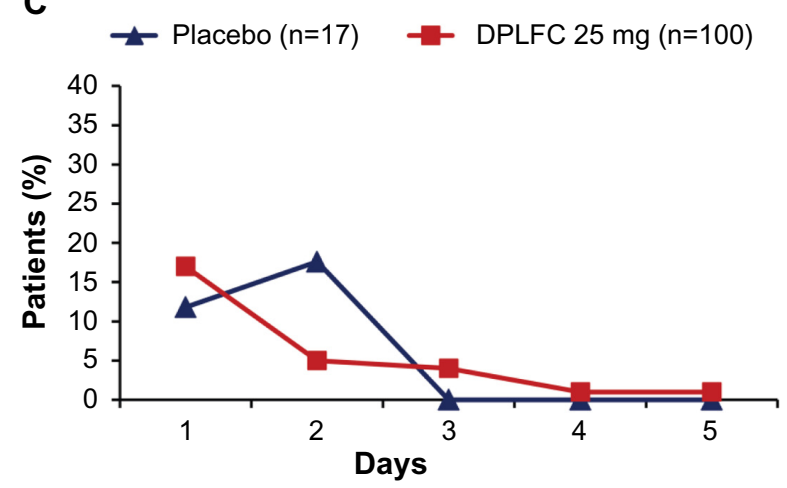

Figure 4 Adverse events collected daily from the time of randomization throughout the study, with the last assessment on day 5.

Notes: The percentage of patients reporting any AE during each day of the study (days I-5) was calculated for all patients in the safety population (A), patients in the safety population taking opioid RM (B), and patients in the safety population not taking opioid RM (C).

Abbreviations: $A E$, adverse event; DPLFC, diclofenac potassium liquid-filled capsule; RM, rescue medication.

likely further reduce the use of opioids and improve the quality of pain management at home.

Treatment of acute pain in the home environment poses various challenges. Patients are more active and are selftreating without the supervision of a health care professional, which may result in increased, inappropriate, and/or prolonged use of analgesics. ${ }^{14,15,31}$ Indeed, in the current analysis, use of both opioid RM and DPLFC increased after discharge compared with the last day before discharge. Importantly, after discharge, patients treated with DPLFC experienced adequate pain relief while still using less opioid
RM than patients treated with placebo. These results show a clinically important opioid-sparing effect of DPLFC in patients self-treating postoperative acute pain under unsupervised conditions, suggesting that when informed about potential benefits of multimodal treatment using an NSAID and an opioid, they may receive better quality treatment in the home environment, minimizing potential opioid misuse and long-term complications. ${ }^{14,31}$

By reducing consumption of opioid RM, DPLFC reduced the incidence of AEs. Most common AEs associated with opioids (nausea, vomiting, dizziness, and constipation) were significantly diminished in patients treated with DPLFC who did not use opioid RM compared with patients who used opioid $\mathrm{RM}$, which is an important clinical benefit. It is important to note, however, that all analgesic agents carry various levels of risk for adverse effects, some potentially serious. NSAIDs are associated with gastrointestinal, cardiovascular, and renal AEs. ${ }^{32}$ Potentially serious AEs of opioids include sedation, respiratory depression, and cognitive and motor impairment, as well as other concerns including dependence and addiction. ${ }^{7,25,33}$ It may be argued that NSAIDs do not incur the same degree of risk as opioids, which are associated with a spectrum of concerns, side effects representing just one of them. Therefore, the potential of NSAIDs such as DPLFC to lower the requirement for opioids while maintaining an adequate analgesic effect offers a range of clinical benefits in the treatment of acute pain., ${ }^{3,10,12}$

\section{Conclusion}

Given the wide spectrum of concerns associated with opioids, including their growing misuse and diversion, better management of acute pain with nonopioid analgesics is of vital importance. Although opioids currently play a leading role in the management of moderate-to-severe pain, NSAIDs (including DPLFC) can provide analgesic benefit, and the multimodal use of NSAIDS and opioids will continue assuming a greater role, especially in the outpatient setting. This is especially important given that patients in the unsupervised environment at home are at higher risk of increased, inappropriate, and/or prolonged use of opioids. No analgesic approach is without risk, but a well-balanced approach using the most effective interventions, while minimizing the risk of harm, will improve recovery profiles for patients with acute pain, including in the home environment.

\section{Disclosure}

IB and SB are employees and shareholders of Depomed, Inc., which funded this research. JSW and NM report no conflicts of interest in this work. 


\section{References}

1. Blondell RD, Azadfard M, Wisniewski AM. Pharmacologic therapy for acute pain. Am Fam Physician. 2013;87(11):766-772.

2. Johnson Q, Borsheski RR, Reeves-Viets JL. Pain management mini-series. Part I. A review of management of acute pain. Mo Med. 2013;110(1):74-79.

3. Nalamachu S. An overview of pain management: the clinical efficacy and value of treatment. Am J Manag Care. 2013;19(Suppl 14):s261-s266.

4. Katz NP, Adams EH, Benneyan JC, et al. Foundations of opioid risk management. Clin J Pain. 2007;23(2):103-118.

5. Gandhi K, Heitz JW, Viscusi ER. Challenges in acute pain management. Anesthesiol Clin. 2011;29(2):291-309.

6. Sinatra R. Causes and consequences of inadequate management of acute pain. Pain Med. 2010;11(12):1859-1871.

7. Dufour R, Joshi AV, Pasquale MK, et al. The prevalence of diagnosed opioid abuse in commercial and Medicare managed care populations. Pain Pract. 2014;14(3):E106-E115.

8. Wightman R, Perrone J, Portelli I, Nelson L. Likeability and abuse liability of commonly prescribed opioids. J Med Toxicol. 2012;8(4): 335-340.

9. Cicero TJ, Ellis MS, Surratt HL, Kurtz SP. Factors influencing the selection of hydrocodone and oxycodone as primary opioids in substance abusers seeking treatment in the United States. Pain. 2013; 154(12):2639-2648.

10. Wong I, St John-Green C, Walker SM. Opioid-sparing effects of perioperative paracetamol and nonsteroidal anti-inflammatory drugs (NSAIDs) in children. Paediatr Anaesth. 2013;23(6):475-495.

11. McDaid C, Maund E, Rice S, Wright K, Jenkins B, Woolacott N. Paracetamol and selective and non-selective non-steroidal antiinflammatory drugs (NSAIDs) for the reduction of morphine-related side effects after major surgery: a systematic review. Health Technol Assess. 2010;14(17):1-153.

12. Reuben SS. Update on the role of nonsteroidal anti-inflammatory drugs and coxibs in the management of acute pain. Curr Opin Anaesthesiol. 2007;20(5):440-450.

13. Rocca GD, Chiarandini P, Pietropaoli P. Analgesia in PACU: nonsteroidal anti-inflammatory drugs. Curr Drug Targets. 2005;6(7):781-787.

14. Srikandarajah S, Gilron I. Systematic review of movement-evoked pain versus pain at rest in postsurgical clinical trials and meta-analyses: a fundamental distinction requiring standardized measurement. Pain. 2011;152(8):1734-1739.

15. Clarke H, Soneji N, Ko DT, Yun L, Wijeysundera DN. Rates and risk factors for prolonged opioid use after major surgery: population based cohort study. BMJ. 2014;348:g1251.

16. Moore N. Diclofenac potassium $12.5 \mathrm{mg}$ tablets for mild to moderate pain and fever: a review of its pharmacology, clinical efficacy and safety. Clin Drug Investig. 2007;27(3):163-195.

17. Cannon CP, Curtis SP, FitzGerald GA, et al. Cardiovascular outcomes with etoricoxib and diclofenac in patients with osteoarthritis and rheumatoid arthritis in the Multinational Etoricoxib and Diclofenac Arthritis Long-term (MEDAL) programme: a randomised comparison. Lancet. 2006;368(9549):1771-1781.

18. Singla NK, Desjardins PJ, Chang PD. A comparison of the clinical and experimental characteristics of four acute surgical pain models: dental extraction, bunionectomy, joint replacement, and soft tissue surgery. Pain. 2014;155(3):441-456.

Journal of Pain Research

\section{Publish your work in this journal}

The Journal of Pain Research is an international, peer-reviewed, open access, online journal that welcomes laboratory and clinical findings in the fields of pain research and the prevention and management of pain. Original research, reviews, symposium reports, hypothesis formation and commentaries are all considered for publication.

Submit your manuscript here: http://www.dovepress.com/journal-of-pain-research-journal
19. Hersh EV, Levin LM, Adamson D, et al. Dose-ranging analgesic study of Prosorb diclofenac potassium in postsurgical dental pain. Clin Ther. 2004;26(8):1215-1227.

20. Zuniga JR, Phillips CL, Shugars D, et al. Analgesic safety and efficacy of diclofenac sodium softgels on postoperative third molar extraction pain. J Oral Maxillofac Surg. 2004;62(7):806-815.

21. Riff DS, Duckor S, Gottlieb I, et al. Diclofenac potassium liquidfilled soft gelatin capsules in the management of patients with postbunionectomy pain: a Phase III, multicenter, randomized, doubleblind, placebo-controlled study conducted over 5 days. Clin Ther. 2009;31(10):2072-2085.

22. Daniels SE, Baum DR, Clark F, Golf MH, McDonnell ME, Boesing SE. Diclofenac potassium liquid-filled soft gelatin capsules for the treatment of postbunionectomy pain. Curr Med Res Opin. 2010;26(10): 2375-2384.

23. Daniels SE, Riff D, Diamond E, Clark F, Boesing SE. An assessment of the efficacy and safety of diclofenac potassium liquid-filled capsules in patients with various levels of baseline pain intensity. Curr Med Res Opin. 2012;28(6):953-961.

24. Mafi JN, McCarthy EP, Davis RB, Landon BE. Worsening trends in the management and treatment of back pain. JAMA Intern Med. 2013;173(17):1573-1581

25. The Joint Commission. Sentinel Event Alert Issue 49: Safe use of opioids in hospitals. Available from: http://www.jointcommission.org/ sea_issue_49/. Accessed November 2, 2014.

26. Centers for Disease Control and Prevention. National Center for Health Statistics Data on Drug Poisoning Deaths. Available from: http://www. cdc.gov/nchs/data/factsheets/factsheet_drug_poisoning.pdf. Accessed November 2, 2014.

27. White PF. The role of non-opioid analgesic techniques in the management of pain after ambulatory surgery. Anesth Analg. 2002;94(3): 577-585.

28. American Society of Anesthesiologists Task Force on Acute Pain Management. Practice guidelines for acute pain management in the perioperative setting: an updated report by the American Society of Anesthesiologists Task Force on Acute Pain Management. Anesthesiology. 2012;116(2):248-273.

29. Maund E, McDaid C, Rice S, Wright K, Jenkins B, Woolacott N. Paracetamol and selective and non-selective non-steroidal antiinflammatory drugs for the reduction in morphine-related side-effects after major surgery: a systematic review. Br J Anaesth. 2011;106(3): 292-297.

30. Ong CK, Seymour RA, Lirk P, Merry AF. Combining paracetamol (acetaminophen) with nonsteroidal antiinflammatory drugs: a qualitative systematic review of analgesic efficacy for acute postoperative pain. Anesth Analg. 2010;110(4):1170-1179.

31. Liu Y, Logan JE, Paulozzi LJ, Zhang K, Jones CM. Potential misuse and inappropriate prescription practices involving opioid analgesics. Am J Manag Care. 2013;19(8):648-665.

32. Fine M. Quantifying the impact of NSAID-associated adverse events. Am J Manag Care. 2013;19(Suppl 14):s267-s272.

33. Liao DL, Huang CY, Hu S, et al. Cognitive control in opioid dependence and methadone maintenance treatment. PLoS One. 2014;9(4):e94589.

The manuscript management system is completely online and includes a very quick and fair peer-review system, which is all easy to use. Visit http://www.dovepress.com/testimonials.php to read real quotes from published authors. 